\title{
Leveraging natural killer cells for cancer immunotherapy
}

\begin{abstract}
Natural killer (NK) cells are potent antitumor effector cells of the innate immune system. Based on their ability to eradicate tumors in vitro and in animal models, significant enthusiasm surrounds the prospect of leveraging human NK cells as vehicles for cancer immunotherapy. While interest in manipulating the effector functions of NK cells has existed for over 30 years, there is renewed optimism for this approach today. Although $\mathrm{T}$ cells receive much of the clinical and preclinical attention when it comes to cancer immunotherapy, new strategies are utilizing adoptive NK-cell immunotherapy and monoclonal antibodies and engineered molecules which have been developed to specifically activate NK cells against tumors. Despite the numerous challenges associated with the preclinical and clinical development of NK cell-based therapies for cancer, NK cells possess many unique immunological properties and hold the potential to provide an effective means for cancer immunotherapy.
\end{abstract}

First draft submitted: 18 January 2017; Accepted for publication: 13 March 2017; Published online: 5 May 2017

Keywords: bortezomib • cancer stem cell $\bullet$ CAR • cetuximab • cytokines $\bullet$ immunotherapy

- lenalidomide $\bullet$ natural killer cell $\bullet$ radiotherapy $\bullet$ rituximab $\bullet$ trastuzumab granzymes, or cytokine secretion, is based on the balance of activating and inhibitory signals received upon target cell engagement $[4,5]$. This natural cytotoxicity and broad target-cell reactivity makes NK cells particularly attractive as vehicles for cancer therapy.

Originally, human NK cells were defined by their ability to spontaneously lyse tumor cells lacking the expression of MHC class I (HLA) without any prior immunization [6]. This phenomenon led to the formation of the 'missing-self hypothesis'. NK cells recognize self-HLA expression on target cells via their killer cell immunoglobulin-like inhibitory receptors (KIRs), allowing them to recognize target cells that have downregulated or altered expression of self [7]. In general, KIR-HLA binding between an NK cell and its target cell leads to the induction of immune tolerance due to the dominant

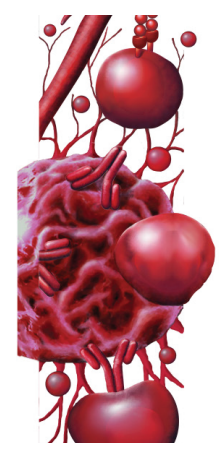

Steven K Grossenbacher ${ }^{1}$, Ethan G Aguilar ${ }^{1}$ \& William J Murphy*,1,2

'Department of Dermatology, University of California Davis School of Medicine, CA 95817, USA

2Department of Internal Medicine, University of California Davis School of Medicine, CA 95817, USA

*Author for correspondence: wmjmurphy@ucdavis.edu
Future : Medicine part of 
negative signaling cascade initiated by the immunoreceptor tyrosine-based inhibition motif found on the intracellular stalk of the KIR protein [8]. However, not all of the proteins within the KIR family are created equal, with KIRs having differential affinity for selfHLA proteins. Additionally, some KIRs are activating instead of inhibitory. KIR expression is variable on a cell-to-cell basis, with KIR genes being expressed stochastically, meaning that within the same individual some NK cells will bind self-HLA with high affinity, while others will lack affinity for self [9]. These differences in KIR expression provide a means for NK-cell 'education' or 'licensing' to occur. NK cells expressing KIRs that are capable of binding self-HLA with high affinity represent the classic cytotoxic NK cells and are thought to be 'licensed to kill' target cells [10]. NK cells expressing KIRs that have lower affinity for self, or lacking expression of KIRs altogether are 'unlicensed' or 'uneducated to self' and are generally hyporesponsive in terms of cytotoxicity compared with their licensed counterpart [10]. This developmental paradigm stratifies NK cells along the spectrum of effector function and maturation, and evidence from human studies suggests that education can have a dramatic impact on NK-cell cytotoxicity toward tumor cells [11].

In addition to KIRs, a variety of other stimulatory and inhibitory cell surface proteins regulate human NK-cell responses and represent targets for NK celldirected therapies. Well studied among these include the NK group 2 member proteins which are both activating (NKG2D and NKG2C) and inhibitory (NKG2A), the Fc receptor CD16, the natural cytotoxicity receptors (NKp30, NKp44, NKp46) and CD244 (2B4) [12]. With the clinical and preclinical development of new immunotherapies for cancer, several other immunoregulatory proteins have also been identified or suggested to influence the effector functions of NK cells [13]. Recent understanding of even more diverse $\mathrm{NK}$-cell subsets has also led to potential changes in how NK cells could be targeted for therapeutic benefit.

Traditionally NK cells were thought to be shortlived effector lymphocytes of the innate immune system, however, recent investigations have identified NKcell populations possessing qualities associated with adaptive immunity, such as long-term persistence and immunological memory [14]. Several groups have identified memory-like and/or adaptive NK cells expressing unique functional and phenotypic characteristics compared with conventional NK cells $[15,16]$. Memorylike and adaptive NK cells appear to be preferentially present in human cytomegalovirus sera-positive individuals and the increased presence of these cells following CMV reactivation in hematopoietic stem cell transplant (HSCT) patients correlates with improved outcomes [17,18]. Interestingly memory-like NK cells have been found to express PD-1, and thus may represent targets for anti-PD-1 checkpoint blockade [19]. The persistence and memory-like features of these subsets of memory-like and adaptive NK cells open up the possibility of isolating more refined populations of effector NK cells that could be exploited, under the right conditions, for cancer immunotherapy.

Despite the fact that in in vitro studies and preclinical models, NK cells are effectively capable of lysing malignant tumor cells; similar efficacy has yet to be fully realized in human clinical trials. However, significant enthusiasm remains over the development of $\mathrm{NK}$ cell-based immunotherapies and several new strategies are being pioneered which may improve the efficacy and application of these cells.

\section{Strategies to engage endogenous NK cells}

NK cells can be activated by a number of different cytokines, including IL-2, IL-12, IL-15, IL-18, IL-21 and type I interferons. IL-2 is the most common of these that has been used to date, with patients routinely receiving IL-2 in combination with NK-cell transfusions to promote cell expansion in vivo [20]. Despite this enhanced expansion, no clinical advantage of the addition of IL-2 therapy was detected in a cohort of patients undergoing autologous transplantation for the treatment of relapsed lymphoma or metastatic breast cancer [21]. A large limitation of IL-2 therapy is the concurrent activation and expansion of $\mathrm{CD} 25+\mathrm{T}$ regulatory (Treg) cells. Tregs express the high affinity IL-2 receptor complex and have been shown to limit NK-cell function not only through IL-2 competition [22,23], but also through TGF- $\beta$-dependent mechanisms [24,25]. Therefore, other cytokines that can more selectively activate NK cells, and not Tregs, are being explored. IL-15 is an essential mediator of NK-cell generation and homeostasis, with previous studies showing that mice deficient in IL-15 or the IL-15 receptor $\alpha$ chain lack mature NK cells [26,27]. A number of recent studies report seeing improved clinical responses correlating with IL-15 treatment or elevated serum IL-15 [28-30]. Recombinant IL-15 administration improved the persistence and proliferation of adoptively transferred haploidentical NK cells in patients with acute myeloid leukemia (AML) [31]. IL-12 is another proinflammatory cytokine with known immunomodulatory properties for NK cells as well as other lymphoid cells. In preclinical models, NK cells have been shown to have enhanced migratory capacity and augmented function following IL-12 administration [32]. To date the use of IL-12 in the clinic has resulted in severe toxicity and limited efficacy, likely due to the pleiotropic nature of IL-12 [33]. Protein and antibody engineering 
has also revealed new methods to improve the pharmacodynamic and pharmacokinetic properties of NK cell-directed therapies. For example, development of so-called 'super agonists' has improved the utility of cytokine-based therapy. The superagonist IL-15-IL$15 \mathrm{R} \alpha-$ Sushi-Fc fusion protein (ALT-803) has been shown to exhibit greater biological activity than native IL-15 and potently stimulate antimetastatic activity from NK cells. Clinical trials are underway to evaluate its safety and efficacy in patients (NCT02099539 and [34]). Combinatorial strategies offer an attractive alternative to these single modality approaches. The combined use of IL-12, IL-15 and IL-18 is one such strategy that has been explored, with data showing this combination induces a long lived cytokine-induced memory-like NK-cell population with enhanced effector functions $[35,36]$.

In addition to cytokines, Toll agonists also play a significant role in NK-cell activation. Human NK cells are thought to express TLRs 1-9 and are responsive to viral and pathogen-associated molecular patterns. Thus the selected utilization of Toll receptor agonists could lead to improved antitumor efficacy via NK-cell activation [37]. Indeed recent experiments have shown that certain synthetic toll agonists are capable of demonstrating antitumor efficacy in murine models of metastatic cancer in an NK cell-dependent manner [38,39].

Several pharmaceutical agents are also potently capable of augmenting NK-cell reactivity toward tumors. The thalidomide derivative lenalidomid $\left(\right.$ Revlimid $\left.^{\circledR}\right)$, is approved in combination with dexamethasone for the treatment of multiple myeloma patients who have received at least one prior therapy. While the precise mechanism of action of lenalidomide is not completely clear, evidence suggests that significant NK-cell immune modulation occurs in response to treatment. For example, lenalidomide is known to increase IL-2 and IFN $\gamma$ secretion from $\mathrm{T}$ cells and dendritic cells, aiding in NK-cell activation and function [40]. Lenalidomid treatment also leads to an increase in circulating NK-cell numbers in leukemia patients [41].

CD16 engagement of an antibody's Fc region is another primary method to activate NK-cell cytotoxicity. Many of the most commercially and clinically successful therapeutic monoclonal antibodies (mAbs), such as rituximab $\left(\operatorname{Retuxin}^{\circledR}\right)$, cetuximab $\left(\right.$ Erbitux $\left.^{\circledR}\right)$ and trastuzumab $\left(\right.$ Herceptin $\left.^{\circledR}\right)$ elicit their therapeutic effects, at least in part, by activating NK cells via antibody-dependent cell-mediated cytotoxicity (ADCC) $[42,43]$. Unlike other activating receptors, which generally require the coordinated engagement of multiple receptors in order to elicit a positive response; CD16 engagement alone is sufficient to provide a robust activation signal for NK cells [44]. For example, the efficacy of anti-GD2 antibody therapy for the treatment of neuroblastoma is achieved through ADCC mediated by NK cells [45]. The interest to generate ADCC-inducing $\mathrm{mAbs}$ has led to the development and engineering of tumor-specific antibodies capable of activating NK cells more robustly than conventional mAbs. Several clinical trials are under way to assess the efficacy of these antibody-based therapies, and new molecules are being designed to engage and/or target multiple stimulatory/inhibitory targets simultaneously [46].

Novel trispecific killer cell engagers and bispecific killer cell engagers have been developed to specifically trigger NK-cell activation by cross-linking tumor antigen-specific antibodies with cytokines such as IL-15, or with antibodies binding to alternative epitopes of CD16 [47-49]. These engineered molecules elicit higher cytotoxic potential compared with conventional antibodies targeting the same antigen [46]. Additionally, CD16 is known to be lost or cleaved from the surface of NK cells rendering them hyporesponsive to ADCC [50]. Based on this finding, strategies are being developed to prevent the cleavage of CD16 through the inhibition of disintegrins and metalloproteases such as ADAM17 [50]. For example, the addition of ADAM17 inhibitors has been found to improve the cytotoxicity of a CD16xCD33 bispecific killer-cell engager against CD33+ AML cells in vitro, and a clinical trial is underway assessing the combination of rituximab with an ADAM17 inhibitor following autologous HSCT for patients with diffuse large B-cell lymphoma (NCT02141451).

Additionally, monoclonal antibodies designed to prevent NK-cell inhibition are in various stages of clinical development. Even though T cells remain the major focus of checkpoint blockade immunotherapy, NK cells have also been found to respond to this approach. NK cells can express PD-1 and a variety of other proteins associated with co-stimulation or inhibition such as TIGIT, Tim-3, CD96, CD137, OX40, GITR and CD27 [51]. The PD-1-PD-L1 pathway is a critical immune regulator limiting the antitumor immune responses of T cells and NK cells. PD-1 is known to be expressed on activated $\mathrm{T}$ cells and was recently found to be expressed on NK cells from patients with multiple myeloma [52]. Interestingly these PD-1+NK cells were capable of killing autologous tumor cells ex vivo, in the presence of an anti-PD-1 antibody, suggesting that PD-1 blockade may augment endogenous NKcell responses by breaking tumor-induced tolerance. It was also recently established that a subset of fully mature, memory-like NK cells expressing PD-1, can have their effector functions augmented in responses 
to anti-PD-1 antibody blockade [19]. In addition to these approaches, KIR blockade has also been evaluated as a method of NK cell-specific checkpoint blockade. Interestingly recent clinical trials have proven the use of a novel anti-KIR2D antagonist (IPH2101) to be ineffective as a monotherapy. In fact IPH2101 treatment led to a rapid reduction in KIR2D expression and rendered NK cells hyporesponsive which may have been due to the antibody blocking the KIRs associated with the education process of NK cells during their development [53]. This study raises concern over the use of conventional antibody-based methods aimed at augmenting endogenous NK-cell responses, particularly through KIR-blockade as this may have unforeseen effects on NK cell development.

\section{Adoptive NK-cell therapy}

The use of unmodified cells either from healthy donors (allogeneic) or cells sourced from the patient themselves (autologous) has been explored for the treatment of a variety of hematological and solid tumor types with varying results [54]. NK cells are well known to be one of the first cell types to reconstitute following HSCT and appear to be a critical mediator of the antitumor efficacy of HSCT [55]. Importantly, patients with quicker NK-cell reconstitution post-transplant have better outcomes [56]. The strongest evidence supporting the efficacy of donor-derived NK cells comes from studies in patients with AML. In the seminal study, patients receiving HLA-haplotype mismatched bone marrow cells with KIR ligand incompatibility were protected from graft rejection, GVHD, and AML relapse when compared with patients who received KIR-ligand compatible cells [57]. Follow-up studies have found similar efficacy for other patients with AML. In the non-HSCT setting, haploidentical NK cells were found to expand in vivo, and led to remission for a subset of high-risk AML patients, with best responses observed in patients who received KIR-mismatched cells [20]. Interestingly, the transfer of purified autologous NK cells into patients with either stage IV renal cell carcinoma or metastatic melanoma led to no meaningful clinical benefits. Despite the lack of clinical benefit in these trials, the transferred NK cells did appear to persist in the patients' circulation for several months, in some cases. It is important to note that the recovered cells expressed lower levels of the activating receptor NKG2D and could not lyse tumor targets in vitro without restimulation with IL-2 [58]. However, it was found that the persistent NK cells could mediate ADCC in vitro, suggesting that combination strategies with monoclonal antibodies may allow for enhanced efficacy of adoptive NK-cell therapy. Additionally, the differences in efficacy of NK-cell therapy between hematological and solid malignancies suggest that approaches which sensitize tumor cells from solid tissues to NK-cell killing, could have significant clinical benefit.

In addition to its direct antitumor effects, ionizing radiotherapy is a potent modulator of tumor cell susceptibility to NK-cell cytotoxicity. The DNA damage response induced by the formation of free radicals in cells exposed to radiotherapy is well known to initiate the transcription of genes associated with cellular stress, such as NKG2D ligands and death receptors, which are recognized by NK cells [59]. Perhaps as a method to restrain the malignant transformation and growth of genotoxically stressed cells, NK cells are intrinsically armed with the ability to identify and lyse stressed targets through their NKG2D activating receptor. In fact, radiotherapy is known to upregulate NKG2D ligands on a variety of cancer cell types in vitro and in vivo leading to increased sensitization to NK-cell-mediated killing [60,61].

Radiotherapy has also been found to enrich for stemlike tumor cells, which can mimic stem cells in their ability to undergo asymmetric differentiation, and have a reduced replicative capacity. Human tumors, especially those arising from solid tissues, are known to have cell-to-cell differences in genetic programs and mutational loads leading to marked intratumoral heterogeneity [62]. This heterogeneity poses a serious problem for the use of conventional chemotherapy, radiotherapy and small molecule drugs aimed at targeting certain genetic drivers and oncogenic pathways within a tumor. So-called 'cancer stem cells' or 'tumor-initiating cells' have the ability to undergo asymmetric cell divisions and are thought to be able to seed relapse and metastasis following conventional therapy [63]. Recent studies have shown that allogeneic and autologous NK cells are inherently capable of recognizing and killing these cancer stem cells in vitro and in vivo. In mouse models of pancreatic cancer, glioblastoma and others, it has been discovered that these stem-like tumor cells express elevated levels of several proteins including MICA, PVR-1 and DR5 that bind to the activating proteins, NKG2D and DNAM-1, and the apoptosisinducing protein, TRAIL, respectively, present on the surface of NK cells, compared with tumor cells with a more differentiated phenotype [64,65]. Similar studies show that radiotherapy, when applied in a rational combination prior to the administration of activated allogeneic NK cell transfer could lead to a synergistic and near complete eradication of cancer stem cells (CSCs) in vivo [60]. This combination strategy could be applied clinically as a way to reduce patient relapse rates and improve the utility of both NK-cell transfer and radiotherapy (Figure 1). NK cells have also been 


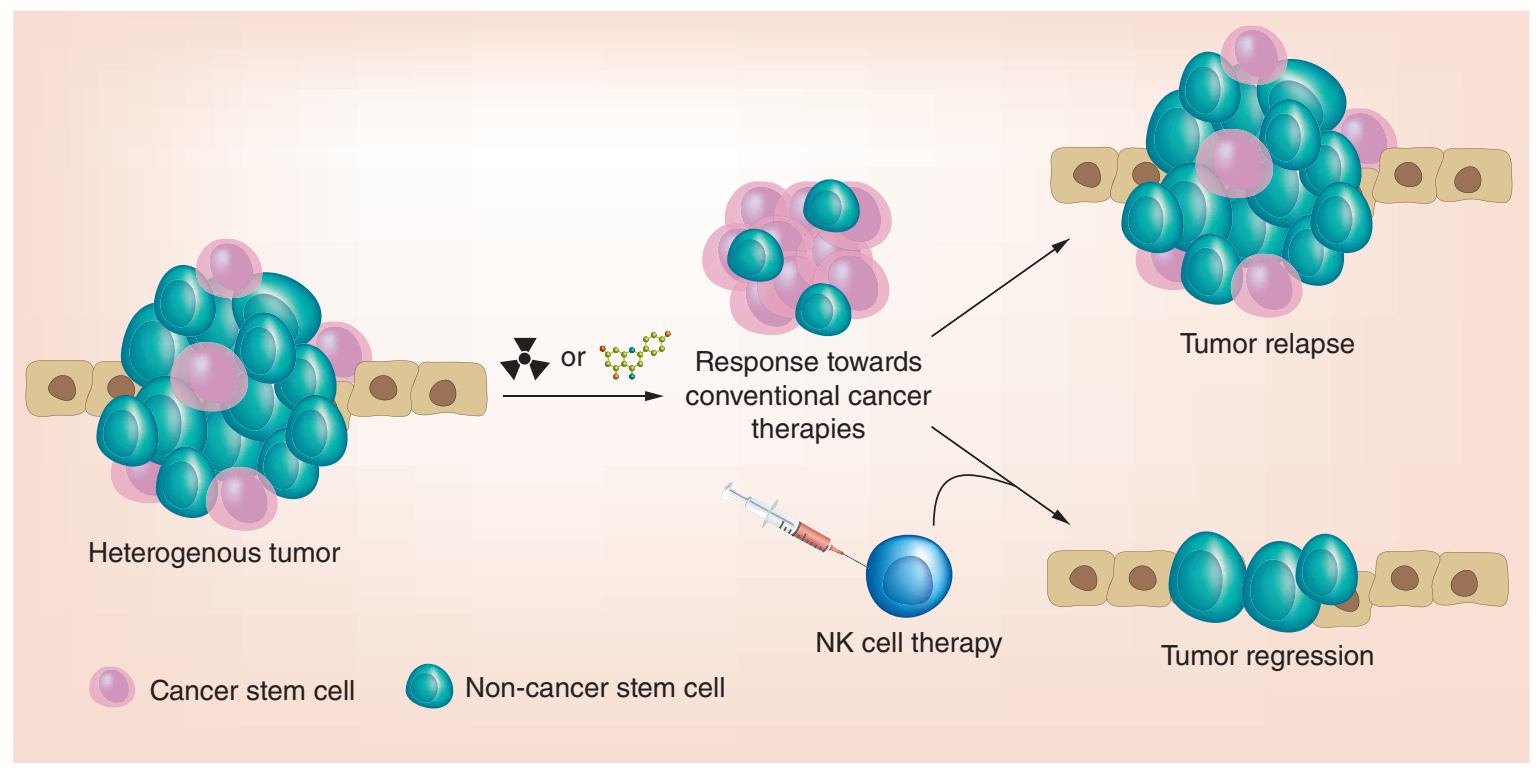

Figure 1. Schematic of natural killer-cell targeting of cancer stem cells following radio/chemotherapy. Populations of cancer stem cells can withstand conventional radiotherapy and/or chemotherapy. In the absence of a cancer stem cell targeting approach, the residual cancer stem cells can seed relapse of the primary tumor. NK cells are capable of killing cancer stem cells and reducing the onset of relapse.

NK: Natural killer.

found to synergize with chemotherapy to target CSCs in preclinical models of nonmuscle invasive bladder cancer [66]. While the role of the cancer stem cell populations in human cancer progression remains controversial, the ability of NK cells to target cancer stem cells may offer a paradigm shift in cancer treatment approaches.

Additionally, the proteasome inhibitor bortezomib (Velcade ${ }^{\circledast}$ ) is known to sensitize tumor cells to NK-cell cytotoxicity by inducing the expression of apoptosisassociated death receptors, such as DR5, and by reducing the generation of peptides normally bound for the HLA complex, leading to a decrease in HLA expression [67-69]. In fact, recent preclinical studies demonstrated that bortezomib is capable of sensitizing tumor cells treated with the oncolytic virus, oHSV-1, to adoptive NK-cell killing [70]. In this study, bortezomib and oHSV-1 synergized to induce the necroptotic cell death of orthotopic glioblastoma xenografts. The addition of intracranially infused NK cells to the combination therapy significantly enhanced tumor cell death leading to enhanced survival of tumor-bearing animals.

Additionally, histone deacytlase inhibition was recently shown to lead to heightened NKG2D expression on pancreatic cancer cells, suggesting that several rational combinations could be increasingly applied with cellular immunotherapy to improve patient outcomes [71,72]. Similar strategies are now being examined to enhance the specificity of adoptive NK-cell therapy through genetic manipulation of NK-cell surface receptors.
Chimeric antigen receptor engineering has transformed the nature of adoptive T-cell therapy. CD19directed $\mathrm{T}$ cells have demonstrated tremendous efficacy in juvenile patients with acute lymphoblastic leukemia [73]. Trials are now underway to assess the efficacy and tolerability of CAR-engineered T cells in a variety of hematological and solid tumors [74]. Despite the interest and clinical success of CAR engineering, CAR-engineered NK cells have yet to make the same imprint on the cancer immunotherapy community. While there are some key advantages for T-cell immunotherapy, CAR-directed NK cells offer an alternative treatment modality that could overcome some of the challenges associated with T-cell CARs, most prominent among them is the manifestation of life-threatening toxicities which have led to the termination of clinical trials and drug development programs [75].

Clinical trials are currently underway investigating the use of CD19-directed NK cells in the treatment of acute lymphoblastic leukemia (Table 1) (NCT00995137 and NCT01974479). The fact that, unlike T cells, NK cells are generally perceived to be short-lived effector cells, which do not undergo clonal expansion, may allow CAR-directed NK cells to be utilized with less fear for the development of autoimmunity or adverse toxicities associated with rampant T-cell expansion that can occur in patients receiving CAR-T. Another concern with the expanded use of CAR-T is the need for the continued and robust expression of a specific antigen. Tumor cells are known to evade immune detection by altering HLA expression 
Table 1. Ongoing clinical trials of natural killer cell adoptive therapy.

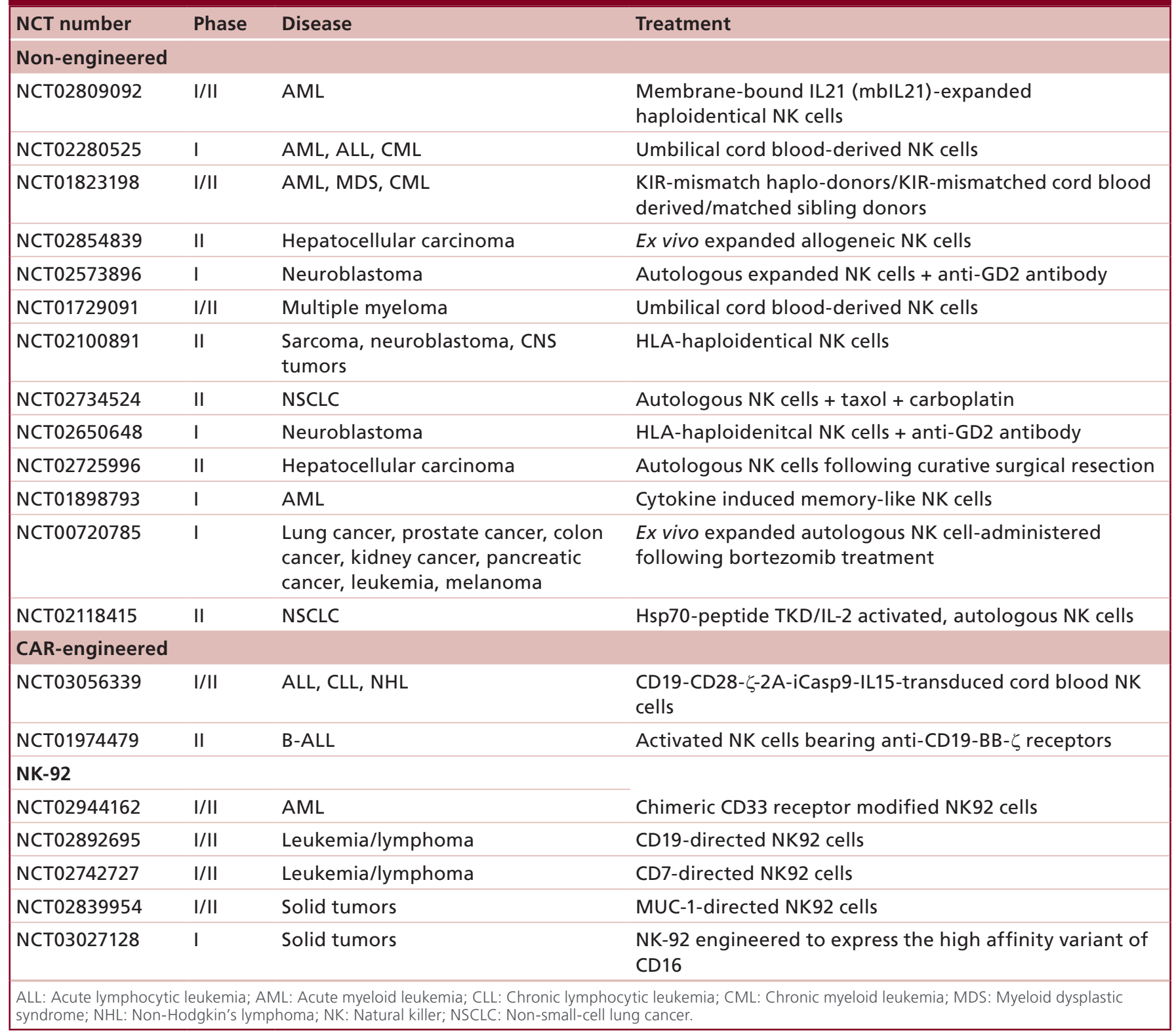

or through tumor heterogeneity and the generation of antigen loss variants [76,77]. Unlike T cells, NK cells kill in an antigen-independent manner and would, therefore, retain mechanisms independent of the CAR to target and kill those tumor cells that would otherwise evade T-cell recognition. Despite the potential benefits of CAR-NK cells, several challenges remain concerning the fact that NK cells can be difficult to manufacture and that different activation and expansion protocols have been found to lead to drastic differences in NK-cell function.

Work is now underway to expand second- and thirdgeneration CAR platforms to include genes which can elicit IL-15 production as well as caspase-controlled suicide vectors in an effort to improve CAR effectiveness and safety [78]. Additionally, the transfection efficiency of NK cells remains fairly low and new methods are being developed in order for CAR-NK technologies to scale-up to the level of CAR-T [79]. An interesting alternative to the use of primary human NK cells is the use of the NK-cell line, NK-92. NK-92 was originally derived from an NK-cell lymphoma and over the years it has consistently shown the ability to be expanded and activated to lyse tumor cell targets since its isolation more than 20 years ago. Because of its ease of use and consistent activity, NK-92 is an attractive platform for CAR development. Preclinical NK-92 cells have been engineered to express a variety of antigen receptors and 
surface proteins including receptors to CD19, HER2 and EGFR [80-82]. Additionally, because NK-92 cells do not express CD16, groups are now working to engineer high affinity CD16 molecules into NK-92 cells as a means to improve their effector functions [83]. As a whole, several clinical trials are underway assessing the efficacy of genetically manipulated autologous NK, allogeneic NK and NK-92 cells for the treatment of hematological and solid malignancies (Table 1). While these strategies have given rise to promising CAR-NK platforms, a key limitation for the use of NK cells for solid tumors is the failure of adoptively transferred NK cells to traffic into tumor sites.

The ability to influence the trafficking and intratumoral homing of a transferred NK-cell population is a significant hurdle for the advancement of adoptive NK-cell therapy. The presence of NK cells in pretreatment tumor biopsies correlates with improved outcomes for patients with solid malignancies [84]; however, the ability of adoptively transferred NK cells to traffic into tumors is severely limited [85]. A fact that may also explain the varied outcomes observed from NK-cell trials in hematological versus solid malignancies. Several strategies are being evaluated which may improve the efficacy of NK-cell therapy by increasing the homing potential of transferred cells into both the tumor site and the draining lymphoid tissues, which may harbor metastatic tumor cells. CCR7-modified NK cells were shown to migrate toward CCL19 and CCL21 in vitro and demonstrated improved lymph node homing in nude mice [86]. Interestingly in these experiments, CCR7 was transferred to the NK cells through the process of trogocytosis. Trogocytosis, the acquisition of cell surface proteins via direct cell-cell contact, has been identified as a means to overcome the challenges associated with viral transfection of primary NK cells. It has been proven as a means to impart functional antigen receptors recognizing CD19 and HER2, as well as chemokine receptors such as CCR7 [87,88]. Despite these increases in NK-cell homing, some have reported that trogocytosis can render immune cells hyporesponsive and act as a method of tumor immune escape [89]. In addition, there are many logistical challenges that would need to be overcome to make the large-scale production of trogocytosis-engineered NK cells feasible. More studies are needed to understand how to overcome the limited ability of NK cells to traffic into solid tumor sites.

A final factor that heavily influences the efficacy, utility and reproducibility of primary NK cells as adoptive therapy is the method of stimulation and expansion. Cytokines such as IL-2, IL-12, IL-15 and IL-18 have been used to induce the activation and expansion of human NK cells in vitro [2]. While cytokine-based activation techniques can yield impressive cytotoxicity in in vitro and in vivo assays, the NK cells become cytokine addicted and quickly lose viability once transferred into the systemic circulation. In addition to cytokines, the use of engineered 'feeder' cell lines has been developed as a method to generate robust NK-cell expansion and activation necessary to meet the needs of clinical utilization. Engineering of cell lines, such as the human K562 cell line with a variety of co-stimulatory proteins including CD137 and membrane forms of IL-15 or IL-21, has been demonstrated to induce greater than 1000-fold expansion of human primary NK cells over a 3- to 4-week period [90]. Continued experimentation is needed to optimize the precise method and cocktail of cytokines and feeder cells capable of maximizing NK cells for clinical use.

\section{Conclusion}

NK cells have proven to be significantly more diverse than originally thought. These stereotypically shortlived effector cells, capable of indiscriminately eliminating tumor cells, are now known to have features of adaptive immunity and may have potential to improve the efficacy of conventional therapies and represent an ideal partner for combinatorial immunotherapy. NK cells could represent a method of immunotherapy that, unlike $\mathrm{T}$ cells, could be more readily fine-tuned to prevent treatment-associated toxicity and immune-mediated adverse events. The development of inexpensive genetic sequencing approaches will allow for further investigation and understanding of how NK-cell education and HLA-KIR interactions affect tumor formation and response to therapy. While murine studies have been the predominant means of examining NK-cell biology, these models are still limited. Inbred mice lack the genetic complexity needed to fully examine the effects of HLAKIR (MHCI-Ly49 in mouse) binding on tumor progression and immune cell function. As a result, new, more complex models are being investigated for the study of immunotherapy, including the use of companion canines bearing spontaneous malignancies, which more closely match the human cancer scenario. The continued genetic manipulation of NK cells into antigen-specific effector cells could lead to improved responses of unmodified cells, however, key obstacles must be considered if NK cell-based immunotherapies are to meet their perceived potential. These obstacles include the ability to traffic into solid tumor sites and avoid tumor immunosuppression, the resistance to current gene transfection techniques, and the development of culture and expansion techniques that promote the reproducible growth and expansion of NK cells for therapy. 


\section{Future perspective}

The clinical success of recent cancer immunotherapies targeting immune checkpoints and utilizing chimeric antigen receptor engineered $T$ cells has ushered in a new age for the treatment of cancer. Immunotherapy now stands as the fourth leg in the clinical arsenal, joining surgery, chemotherapy and radiotherapy, to treat a variety of hematological and solid malignancies. The key for the continued success of cancer immunotherapy will be to understand how different combinatorial strategies can be harnessed and tailored to meet the needs of certain patient populations. An in-depth understanding of the antitumor efficacy and function of NK cells will allow future clinicians and scientists to better leverage the innate killing abilities of these cells. While it is unlikely that unmodified NK cells themselves can be completely efficacious as a monotherapy, it is likely that certain tumor types will respond favorably to NK-cell treatment in combination with other therapies. Those tumors harboring more undifferentiated cell types, and expressing higher levels of stress-associated and/or stem-like proteins, would be particular good candidates for NK-cell therapy in combination with conventional chemotherapy and/or radiotherapy. Last, as we develop a better understanding of both the physiological relationship between CMV exposure and NK-cell functions, and the immunological consequences of NK-cell education, tumor immunologists will be able to shed light on how early tumorigenesis occurs in spite of the innate recognition and killing functions that are inherent to NK cells. Therapeutic strategies designed to leverage NK cells will continue to be part of the cancer immunotherapy paradigm for years to come.

\section{Financial \& competing interests disclosure}

This work was supported by the NIH/National Cancer Institute award R01 CA189209. The authors have no other relevant affiliations or financial involvement with any organization or entity with a financial interest in or financial conflict with the subject matter or materials discussed in the manuscript apart from those disclosed.

No writing assistance was utilized in the production of this manuscript.

\section{Executive summary}

- Natural killer (NK) cells are innate lymphocytes found in the peripheral circulation and in lymphoid tissues. NK cells are capable of lysing virally infected and malignantly transformed cells without prior immunization or exposure. NK-cell effector functions are regulated by the balance of activating and inhibitory signals received from cell surface receptors and proteins upon encountering a target cell.

- NK cells exist as diverse populations with different phenotypes and propensities for cytotoxicity and cytokine function. This diversity is the result of CMV exposure and influences NK-cell cytotoxicity of tumor cells.

- NK cells are known to express a variety of co-stimulatory molecules which represent targets for novel immunotherapies. Recent clinical trials investigating the use of inhibitory KIR blockade proved ineffective as a monotherapy.

- NK cells are particularly effective at recognizing and killing cancer stem cells. Following conventional therapy, such as radiotherapy and/or chemotherapy, NK cells are able to identify residual tumor cells and lyse them via NKG2D and other activating receptors.

- CAR-NK-cell therapy may be more advantageous than T-cell CARs due to limited clonal expansion, leading to reduced toxicity. However, the transfection techniques and methods of isolation and expansion of cells ex vivo can affect the final cell therapy dramatically.

- All of these data suggest that combinatorial strategies may be beneficial for leveraging NK cells for cancer immunotherapy.

\section{References}

1 Cerwenka A, Lanier LL. Natural killer cell memory in infection, inflammation and cancer. Nat. Rev. Immunol. 16(2), 112-123 (2016).

2 Guillerey C, Huntington ND, Smyth MJ. Targeting natural killer cells in cancer immunotherapy. Nat. Immunol. 17(9), 1025-1036 (2016).

3 Vivier E, Tomasello E, Baratin M, Walzer T, Ugolini S. Functions of natural killer cells. Nat. Immunol. 9(5), 503-510 (2008).

4 Morvan MG, Lanier LL. NK cells and cancer: you can teach innate cells new tricks. Nat. Rev. Cancer 16(1), 7-19 (2016).

5 Dahlberg CI, Sarhan D, Chrobok M, Duru AD, Alici E. Natural killer cell-based therapies targeting cancer: possible strategies to gain and sustain anti-tumor activity. Front. Immunol. 6, 605 (2015).

6 Kumar V, Mcnerney ME. A new self: MHC-class-Iindependent natural-killer-cell self-tolerance. Nat. Rev. Immunol. 5(5), 363-374 (2005).

7 Pegram HJ, Andrews DM, Smyth MJ, Darcy PK, Kershaw $\mathrm{MH}$. Activating and inhibitory receptors of natural killer cells. Immunol. Cell Biol. 89(2), 216-224 (2011). 
8 Long EO. Negative signaling by inhibitory receptors: the NK cell paradigm. Immunol. Rev. 224, 70-84 (2008).

9 Husain Z, Alper CA, Yunis EJ, Dubey DP. Complex expression of natural killer receptor genes in single natural killer cells. Immunology 106(3), 373-380 (2002).

10 Kim S, Poursine-Laurent J, Truscott SM et al. Licensing of natural killer cells by host major histocompatibility complex class I molecules. Nature 436(7051), 709-713 (2005).

11 Tarek N, Le Luduec JB, Gallagher MM et al. Unlicensed NK cells target neuroblastoma following anti-GD2 antibody treatment. J. Clin. Invest. 122(9), 3260-3270 (2012).

12 Long EO, Kim HS, Liu D, Peterson ME, Rajagopalan S. Controlling natural killer cell responses: integration of signals for activation and inhibition. Annu. Rev. Immunol. 31, 227-258 (2013).

13 Shevtsov M, Multhoff G. Immunological and translational aspects of NK cell-based antitumor immunotherapies. Front. Immunol. 7, 492 (2016).

14 Sun JC, Beilke JN, Lanier LL. Adaptive immune features of natural killer cells. Nature 457(7229), 557-561 (2009).

15 Schlums H, Cichocki F, Tesi B et al. Cytomegalovirus infection drives adaptive epigenetic diversification of NK cells with altered signaling and effector function. Immunity 42(3), 443-456 (2015).

16 Van Den Boorn JG, Jakobs C, Hagen C et al. Inflammasome-dependent induction of adaptive NK cell memory. Immunity 44(6), 1406-1421 (2016).

17 Cichocki F, Cooley S, Davis Z et al. CD56dimCD57+NKG2C+ NK cell expansion is associated with reduced leukemia relapse after reduced intensity HCT. Leukemia 30 (2), 456-463 (2016).

18 Lee J, Zhang T, Hwang I et al. Epigenetic modification and antibody-dependent expansion of memory-like NK cells in human cytomegalovirus-infected individuals. Immunity 42(3), 431-442 (2015).

19 Della Chiesa M, Pesce S, Muccio L et al. Features of memory-like and PD-1(+) human NK cell subsets. Front. Immunol. 7, 351 (2016).

20 Miller JS, Soignier Y, Panoskaltsis-Mortari A et al. Successful adoptive transfer and in vivo expansion of human haploidentical NK cells in patients with cancer. Blood 105(8), 3051-3057 (2005)

21 Burns LJ, Weisdorf DJ, Defor TE et al. IL-2-based immunotherapy after autologous transplantation for lymphoma and breast cancer induces immune activation and cytokine release: a Phase I/II trial. Bone Marrow Transplant. 32(2), 177-186 (2003)

22 Gasteiger G, Hemmers S, Bos PD, Sun JC, Rudensky AY. IL2-dependent adaptive control of NK cell homeostasis. J. Exp. Med. 210(6), 1179-1187 (2013).

23 Gasteiger G, Hemmers S, Firth MA et al. IL-2-dependent tuning of NK cell sensitivity for target cells is controlled by regulatory T cells. J. Exp. Med. 210(6), 1167-1178 (2013).

24 Ghiringhelli F, Menard C, Terme M et al. CD4+CD25+ regulatory $\mathrm{T}$ cells inhibit natural killer cell functions in a transforming growth factor-beta-dependent manner. J. Exp. Med. 202(8), 1075-1085 (2005).
25 Smyth MJ, Teng MW, Swann J, Kyparissoudis K, Godfrey DI, Hayakawa Y. CD4+CD25+ T regulatory cells suppress NK cell-mediated immunotherapy of cancer. J. Immunol. 176(3), 1582-1587 (2006).

26 Lodolce JP, Boone DL, Chai S et al. IL-15 receptor maintains lymphoid homeostasis by supporting lymphocyte homing and proliferation. Immunity 9(5), 669-676 (1998).

27 Kennedy MK, Glaccum M, Brown SN et al. Reversible defects in natural killer and memory CD8 T cell lineages in interleukin 15-deficient mice. J. Exp. Med. 191(5), 771-780 (2000).

28 Porrata LF, Inwards DJ, Micallef IN et al. Interleukin-15 affects patient survival through natural killer cell recovery after autologous hematopoietic stem cell transplantation for non-Hodgkin lymphomas. Clin. Dev. Immunol. 2010, 914945 (2010).

29 Conlon KC, Lugli E, Welles HC et al. Redistribution, hyperproliferation, activation of natural killer cells and CD8 $\mathrm{T}$ cells, and cytokine production during first-in-human clinical trial of recombinant human interleukin-15 in patients with cancer. J. Clin. Oncol. 33(1), 74-82 (2015).

30 Perez-Martinez A, Fernandez L, Valentin J et al. A Phase I/II trial of interleukin-15-stimulated natural killer cell infusion after haplo-identical stem cell transplantation for pediatric refractory solid tumors. Cytotherapy 17(11), 1594-1603 (2015).

31 Cooley S, Verneris MR, Curtsinger J et al. Recombinant human IL-15 promotes in vivo expansion of adoptively transferred NK cells in a first-in-human Phase I dose escalation study in patients with AML. Blood $120(21), 894$ (2012).

32 Allavena P, Paganin C, Zhou D, Bianchi G, Sozzani S, Mantovani A. Interleukin-12 is chemotactic for natural killer cells and stimulates their interaction with vascular endothelium. Blood 84(7), 2261-2268 (1994).

33 Gollob JA, Mier JW, Veenstra K et al. Phase I trial of twiceweekly intravenous interleukin 12 in patients with metastatic renal cell cancer or malignant melanoma: ability to maintain IFN-gamma induction is associated with clinical response. Clin. Cancer Res. 6(5), 1678-1692 (2000).

34 Kim PS, Kwilas AR, Xu W et al. IL-15 superagonist/IL15RalphaSushi-Fc fusion complex (IL-15SA/IL-15RalphaSuFc; ALT-803) markedly enhances specific subpopulations of $\mathrm{NK}$ and memory CD8 + T cells, and mediates potent antitumor activity against murine breast and colon carcinomas. Oncotarget 7(13), 16130-16145 (2016).

35 Cooper MA, Elliott JM, Keyel PA, Yang L, Carrero JA, Yokoyama WM. Cytokine-induced memory-like natural killer cells. Proc. Natl Acad Sci. USA 106(6), 1915-1919 (2009).

36 Romee R, Rosario M, Berrien-Elliott MM et al. Cytokineinduced memory-like natural killer cells exhibit enhanced responses against myeloid leukemia. Sci. Transl. Med. 8(357), 357ra123 (2016).

37 Adib-Conquy M, Scott-Algara D, Cavaillon JM, SouzaFonseca-Guimaraes F. TLR-mediated activation of NK cells and their role in bacterial/viral immune responses in mammals. Immunol. Cell Biol. 92(3), 256-262 (2014). 
38 Brackett CM, Kojouharov B, Veith J et al. Toll-like receptor-5 agonist, entolimod, suppresses metastasis and induces immunity by stimulating an NK-dendritic-CD8+ T-cell axis. Proc. Natl Acad Sci. USA 113(7), E874-883 (2016).

39 Yang H, Brackett CM, Morales-Tirado VM et al. The Toll-like receptor 5 agonist entolimod suppresses hepatic metastases in a murine model of ocular melanoma via an NK cell-dependent mechanism. Oncotarget 7(3), 2936-2950 (2016).

40 Reddy N, Hernandez-Ilizaliturri FJ, Deeb G et al. Immunomodulatory drugs stimulate natural killer-cell function, alter cytokine production by dendritic cells, and inhibit angiogenesis enhancing the anti-tumour activity of rituximab in vivo. Br. J. Haematol. 140 (1), 36-45 (2008).

41 Chanan-Khan AA, Chitta K, Ersing N et al. Biological effects and clinical significance of lenalidomide-induced tumour flare reaction in patients with chronic lymphocytic leukaemia: in vivo evidence of immune activation and antitumour response. Br. J. Haematol. 155(4), 457-467 (2011).

42 Wang W, Erbe AK, Hank JA, Morris ZS, Sondel PM. NK Cell-mediated antibody-dependent cellular cytotoxicity in cancer immunotherapy. Front. Immunol. 6, 368 (2015).

43 Seidel UJ, Schlegel P, Lang P. Natural killer cell mediated antibody-dependent cellular cytotoxicity in tumor immunotherapy with therapeutic antibodies. Front. Immunol. 4, 76 (2013)

44 Mandelboim O, Malik P, Davis DM, Jo CH, Boyson JE, Strominger JL. Human CD16 as a lysis receptor mediating direct natural killer cell cytotoxicity. Proc. Natl Acad Sci. USA 96(10), 5640-5644 (1999).

45 Sondel PM, Hank JA. Combination therapy with interleukin-2 and antitumor monoclonal antibodies. Cancer J. Sci. Am. 3(Suppl. 1), S121-127 (1997).

46 Gleason MK, Verneris MR, Todhunter DA et al. Bispecific and trispecific killer cell engagers directly activate human NK cells through CD16 signaling and induce cytotoxicity and cytokine production. Mol. Cancer Ther. 11(12), 2674-2684 (2012).

47 Schmohl JU, Felices M, Taras E, Miller JS, Vallera DA. Enhanced ADCC and NK cell activation of an anticarcinoma bispecific antibody by genetic insertion of a modified IL-15 cross-linker. Mol. Ther. 24(7), 1312-1322 (2016).

48 Vallera DA, Felices M, Mcelmurry R et al. IL15 trispecific killer engagers (TriKE) make natural killer cells specific to $\mathrm{CD} 33$ + targets while also inducing persistence, in vivo expansion, and enhanced function. Clin. Cancer Res. 22(14), 3440-3450 (2016).

49 Gleason MK, Ross JA, Warlick ED et al. CD16xCD33 bispecific killer cell engager (BiKE) activates NK cells against primary MDS and MDSC CD33+ targets. Blood 123(19), 3016-3026 (2014).

50 Romee R, Foley B, Lenvik T et al. NK cell CD16 surface expression and function is regulated by a disintegrin and metalloprotease-17 (ADAM17). Blood 121(18), 3599-3608 (2013).
51 Chester C, Fritsch K, Kohrt HE. Natural killer cell immunomodulation: targeting activating, inhibitory, and co-stimulatory receptor signaling for cancer immunotherapy. Front. Immunol. 6, 601 (2015).

52 Benson DM Jr, Bakan CE, Mishra A et al. The PD-1/PD-L1 axis modulates the natural killer cell versus multiple myeloma effect: a therapeutic target for CT-011, a novel monoclonal anti-PD-1 antibody. Blood 116(13), 2286-2294 (2010).

53 Carlsten M, Korde N, Kotecha R et al. Checkpoint inhibition of KIR2D with the monoclonal antibody IPH2101 induces contraction and hyporesponsiveness of NK cells in patients with myeloma. Clin. Cancer Res. 22(21), 5211-5222 (2016).

54 Childs RW, Carlsten M. Therapeutic approaches to enhance natural killer cell cytotoxicity against cancer: the force awakens. Nat. Rev. Drug Discov. 14(7), 487-498 (2015).

55 Seggewiss R, Einsele H. Immune reconstitution after allogeneic transplantation and expanding options for immunomodulation: an update. Blood 115(19), 3861-3868 (2010).

56 Savani BN, Mielke S, Adams S et al. Rapid natural killer cell recovery determines outcome after T-cell-depleted HLAidentical stem cell transplantation in patients with myeloid leukemias but not with acute lymphoblastic leukemia. Leukemia 21(10), 2145-2152 (2007).

57 Ruggeri L, Capanni M, Urbani E et al. Effectiveness of donor natural killer cell alloreactivity in mismatched hematopoietic transplants. Science 295(5562), 2097-2100 (2002).

58 Parkhurst MR, Riley JP, Dudley ME, Rosenberg SA. Adoptive transfer of autologous natural killer cells leads to high levels of circulating natural killer cells but does not mediate tumor regression. Clin. Cancer Res. 17(19), 6287-6297 (2011).

59 Kim JY, Son YO, Park SW et al. Increase of NKG2D ligands and sensitivity to NK cell-mediated cytotoxicity of tumor cells by heat shock and ionizing radiation. Exp. Mol. Med. 38(5), 474-484 (2006).

60 Ames E, Canter RJ, Grossenbacher SK et al. Enhanced targeting of stem-like solid tumor cells with radiation and natural killer cells. Oncoimmunology 4(9), e1036212 (2015).

61 Gasser S, Orsulic S, Brown EJ, Raulet DH. The DNA damage pathway regulates innate immune system ligands of the NKG2D receptor. Nature 436(7054), 1186-1190 (2005).

62 Gerlinger M, Rowan AJ, Horswell S et al. Intratumor heterogeneity and branched evolution revealed by multiregion sequencing. N. Engl. J. Med. 366(10), 883-892 (2012).

63 Wicha MS, Liu S, Dontu G. Cancer stem cells: an old idea a paradigm shift. Cancer Res. 66(4), 1883-1890, discussion 1895-1886 (2006).

64 Ames E, Canter RJ, Grossenbacher SK et al. NK cells preferentially target tumor cells with a cancer stem cell phenotype. J. Immunol. 195(8), 4010-4019 (2015).

65 Castriconi R, Daga A, Dondero A et al. NK cells recognize and kill human glioblastoma cells with stem cell-like properties. J. Immunol. 182(6), 3530-3539 (2009).

66 Ferreira-Teixeira M, Paiva-Oliveira D, Parada B et al. Natural killer cell-based adoptive immunotherapy eradicates and 
drives differentiation of chemoresistant bladder cancer stemlike cells. BMC Med. 14(1), 163 (2016).

terte A, Berg M, Smith A, Childs RW. Bortezomib treatment to potentiate the anti-tumor immunity of ex-vivo expanded adoptively infused autologous natural killer cells. $J$. Cancer 2, 383-385 (2011).

68 Lundqvist A, Su S, Rao S, Childs R. Cutting edge: bortezomib-treated tumors sensitized to NK-cell apoptosis paradoxically acquire resistance to antigen-specific T cells. J. Immunol. 184(3), 1139-1142 (2010).

69 Hallett WH, Ames E, Motarjemi M et al. Sensitization of tumor cells to NK cell-mediated killing by proteasome inhibition. J. Immunol. 180(1), 163-170 (2008).

70 Yoo JY, Jaime-Ramirez AC, Bolyard C et al. Bortezomib treatment sensitizes oncolytic HSV-1-treated tumors to NKcell immunotherapy. Clin. Cancer Res. 22(21), 5265-5276 (2016).

71 Zhu S, Denman CJ, Cobanoglu ZS et al. The narrowspectrum HDAC inhibitor entinostat enhances NKG2D expression without NK-cell toxicity, leading to enhanced recognition of cancer cells. Pharm. Res. 32(3), 779-792 (2015).

72 Diermayr S, Himmelreich H, Durovic B et al. NKG2D ligand expression in AML increases in response to HDAC inhibitor valproic acid and contributes to allorecognition by NK-cell lines with single KIR-HLA class I specificities. Blood 111(3), 1428-1436 (2008).

73 Maude SL, Teachey DT, Porter DL, Grupp SA. CD19targeted chimeric antigen receptor T-cell therapy for acute lymphoblastic leukemia. Blood 125(26), 4017-4023 (2015).

74 Ramos CA, Savoldo B, Dotti G. CD19-CAR trials. Cancer J. 20(2), 112-118 (2014).

75 Brudno JN, Kochenderfer JN. Toxicities of chimeric antigen receptor T cells: recognition and management. Blood 127(26), 3321-3330 (2016).

76 Beatty GL, Gladney WL. Immune escape mechanisms as a guide for cancer immunotherapy. Clin. Cancer Res. 21(4), 687-692 (2015).

77 Khong HT, Restifo NP. Natural selection of tumor variants in the generation of 'tumor escape' phenotypes. Nat. Immunol. 3(11), 999-1005 (2002).

78 Sahm C, Schonfeld K, Wels WS. Expression of IL-15 in NK cells results in rapid enrichment and selective cytotoxicity of gene-modified effectors that carry a tumor-specific antigen receptor. Cancer Immunol. Immunother. 61(9), 1451-1461 (2012).

79 Carlsten M, Childs RW. Genetic manipulation of NK cells for cancer immunotherapy: techniques and clinical implications. Front. Immunol. 6, 266 (2015).

80 Romanski A, Uherek C, Bug G et al. CD19-CAR engineered NK-92 cells are sufficient to overcome NK cell resistance in B-cell malignancies. J. Cell. Mol. Med. 20(7), 1287-1294 (2016).

81 Han J, Chu J, Keung Chan W et al. CAR-engineered NK cells targeting wild-type EGFR and EGFRvIII enhance killing of glioblastoma and patient-derived glioblastoma stem cells. Sci. Rep. 5, 11483 (2015).

82 Schonfeld K, Sahm C, Zhang C et al. Selective inhibition of tumor growth by clonal NK cells expressing an ErbB2/ HER2-specific chimeric antigen receptor. Mol. Ther. 23(2), 330-338 (2015).

83 Jochems C, Hodge JW, Fantini M et al. An NK cell line (haNK) expressing high levels of granzyme and engineered to express the high affinity CD16 allele. Oncotarget 7(52), 86359-86373 (2016).

84 Mamessier E, Sylvain A, Thibult ML et al. Human breast cancer cells enhance self tolerance by promoting evasion from NK cell antitumor immunity. J. Clin. Invest. 121(9), 3609-3622 (2011).

85 Melero I, Rouzaut A, Motz GT, Coukos G. T-cell and NK-cell infiltration into solid tumors: a key limiting factor for efficacious cancer immunotherapy. Cancer Discov. 4(5), 522-526 (2014).

86 Somanchi SS, Somanchi A, Cooper LJ, Lee DA. Engineering lymph node homing of ex vivo-expanded human natural killer cells via trogocytosis of the chemokine receptor CCR7. Blood 119(22), 5164-5172 (2012).

87 Suzuki E, Kataoka TR, Hirata M et al. Trogocytosismediated expression of HER 2 on immune cells may be associated with a pathological complete response to trastuzumab-based primary systemic therapy in HER2overexpressing breast cancer patients. BMC Cancer 15, 39 (2015).

88 Cho FN, Chang TH, Shu CW et al. Enhanced cytotoxicity of natural killer cells following the acquisition of chimeric antigen receptors through trogocytosis. PLoS One 9(10), e109352 (2014).

89 Miner CA, Giri TK, Meyer CE, Shabsovich M, Tripathy SK. Acquisition of activation receptor ligand by trogocytosis renders NK cells hyporesponsive. J. Immunol. 194(4), 1945-1953 (2015).

90 Denman CJ, Senyukov VV, Somanchi SS et al. Membranebound IL-21 promotes sustained ex vivo proliferation of human natural killer cells. PLoS One 7(1), e30264 (2012). 
